\title{
POISSONIAN AND LAPLACIAN FIELD ANALOGUE FOR THE SOLUTION OF TWO DIMENSIONAL COMPLEX HEAT CONDUCTION PROBLEM
}
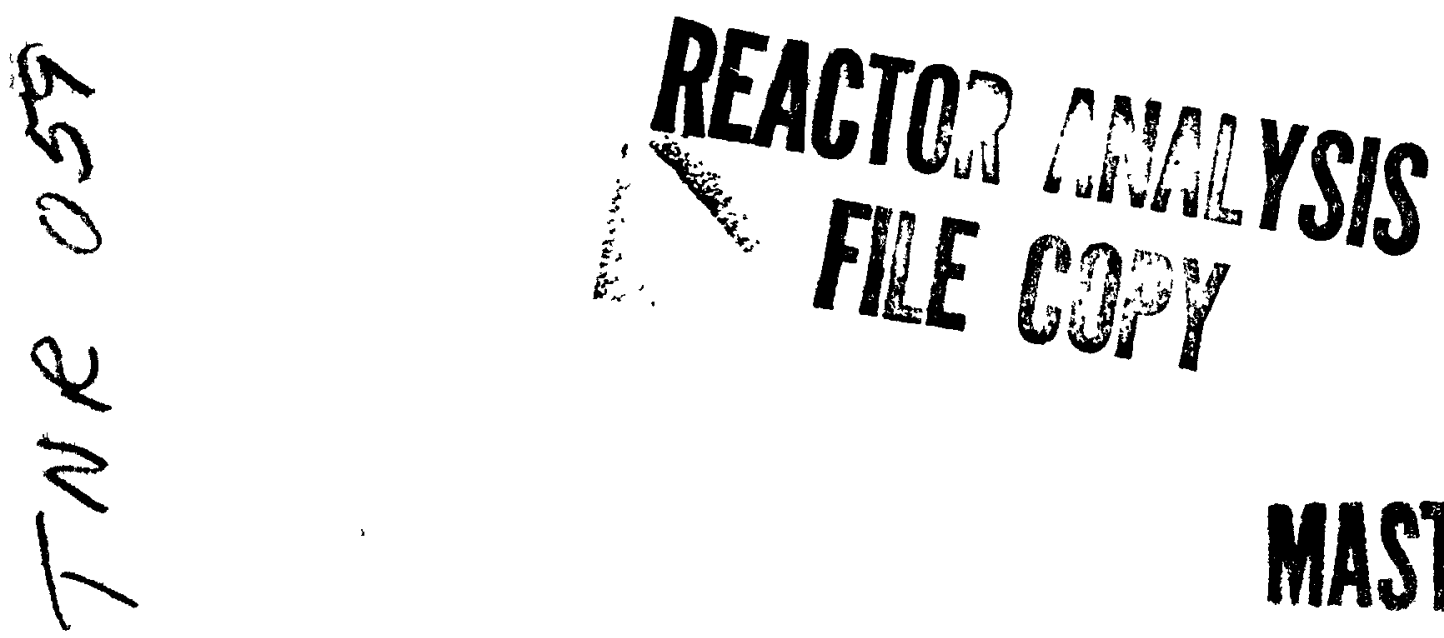

\section{MASTER}

\section{Astronuclear Laboratory \\ Westinghouse Electric Corporation}




\section{DISCLAIMER}

This report was prepared as an account of work sponsored by an agency of the United States Government. Neither the United States Government nor any agency Thereof, nor any of their employees, makes any warranty, express or implied, or assumes any legal liability or responsibility for the accuracy, completeness, or usefulness of any information, apparatus, product, or process disclosed, or represents that its use would not infringe privately owned rights. Reference herein to any specific commercial product, process, or service by trade name, trademark, manufacturer, or otherwise does not necessarily constitute or imply its endorsement, recommendation, or favoring by the United States Government or any agency thereof. The views and opinions of authors expressed herein do not necessarily state or reflect those of the United States Government or any agency thereof. 


\section{DISCLAIMER}

Portions of this document may be illegible in electronic image products. Images are produced from the best available original document. 
WANL-TNR-059

C. 24

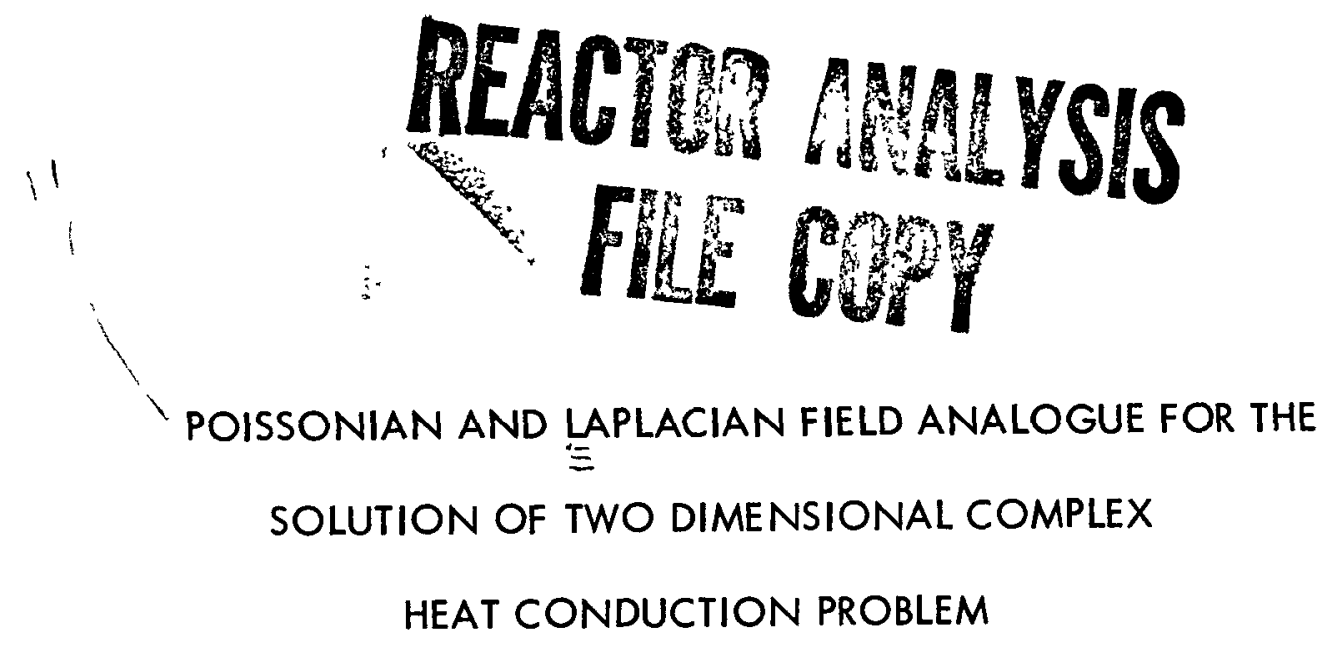

on

By: B. L. Pierce

ASTRONUCLEAR LABORATORY

WESTINGHOUSE ELECTRIC CORPORATION

June, 1962 
TABLE OF CONTENTS

List of Figures

Abstract

iii

Nomenclature

iv

1.0 Introduction

2.0 Continuous Resistor (PAL Type 1) Analogue

2

3.0 Lumped Resistor (PAL Type 2) Analogue

8

4.0 Standard Resistance

5.0 Difference in Thermal Conductivity Between Regions 15

6.0 Non-isothermal Boundary Conditions 16

7.0 Conclusions 16

8.0 References 18

Appendix A Derivation of the Temperature Difference Equation

Appendix B Temperature Difference Equation for an Infinite Plane

Plate with Linear Heat Generation

Appendix C

Figures

23-30 


\section{LIST OF FIGURES}

Figure

Page

Examples of Analogies Between Problems in Five

Different Types of Laplacian Fields

Type 1) Analogue

(2a $\times 2 a)$ with Uniform Heat Generation (PAL Analogue

\section{Type 1)}

4

Circuit Diagram of a Lumped Resistor (PAL Analogue Type 2)

Analogue

(2a $\times 2 a)$ with Uniform Heat Generation (PAL Analogue

Type 2) $y=a$

(2a $\times 2 a)$ with Uniform Heat Generation (PAL Analogue

Type 2) $x=a$

(2a $\times 2 a)$ with Uniform Heat Generation (PAL Analogue

Type 2) $x=y$ 


\begin{abstract}
This report presents the theory, possible applications and accuracy of the Poissonian and Laplacian fields analogue (PAL analogue). The PAL analogue determines the two dimensional equipotential (isothermal) patterns of Poissonian fields created by uniform on non-uniform sources (heat generation). The analytical results of a number of steady-state problems are compared with the results from the PAL analogue to demonstrate its accuracy.
\end{abstract}




\section{NOMENCLATURE}

\begin{tabular}{|c|c|}
\hline$a_{p} l, L$ & -length \\
\hline$x, y, \alpha, \rho$ & -orthogonal coordinates \\
\hline A & -area \\
\hline$T$ & -Temperature \\
\hline$E$ & -voltage \\
\hline$q^{\prime \prime \prime}$ & -heat generation \\
\hline$i^{\prime \prime \prime}$ & -current flux \\
\hline k & -conductivity \\
\hline$h$ & -film heat transfer coefficient \\
\hline$Q$ & -total heat \\
\hline I & -total current \\
\hline$f, F$ & -function \\
\hline$c_{1}, c_{2}$ & -constants \\
\hline SUBSCRIPT & \\
\hline t & -thermal \\
\hline e & -electrical \\
\hline std & -standard \\
\hline & -wall \\
\hline
\end{tabular}




\subsection{INTRODUCTION}

In the field of nuclear heat transfer many problems involving Poissonian fields and encountered. Only for the simplest geometric shapes and boundary conditions are analytical solutions known for this type of problem. The PAL analogue supplies the need for a quick, easy and inexpensive method of solving these problems with complicated geometrical shapes and boundary conditions.

The PAL analogue is a geometrically simi lar analogue that uses a conducting paper that has geometric similarity to the body being investigated. This type of analogue can be sub-classified into two categories.

1. Absolute electrical similarity (PAL analogue Type 1)

2. Modified electrical similarity (PAL analogue Type 2)

The PAL analogue Type 1 restricts the manner in which the electric current can be introduced because of the absolute geometric and electric similarity. This type of analogue requires that the current enter the analogue continuously over an area that represents the particular problem being investigated.

The first method of uniformly introducing a current throughout an area of conducting paper consists of bonding the paper directly to a slab of material of high electrical resistivity. The electric current introduced into the conducting paper in this manner is analogous to heat generation which it simulates. This method is applicable to steadystate heat conduction problems with uniform heat generation.

The PAL analogue Type 2 has geometric similarity with the problem but not with respect to the heat generation. The electric current, which represents the heat 
generated throughout some area, is introduced at grid points instead of continuously. The current, therefore, enters the analogue in a lumped manner that is not identically analogous to the heat generation. The heat flow pattern is approximated very closely if a sufficiently fine matrix is used. This analogue has the disadvantage of lumping but has the advantage over the Type 1 of its ability to handle problems that involve heat generation which varies with regard to geometry.

The numerous electrical connections that are required are made accurately and rapidly with the aid of a specially designed grid board.

Both types of the PAL analogue use an electrically conducting paper. This paper ("Teledeltos") can be easily formed into complicated two dimensional shapes and allows the means of applying the proper boundary conditions. The "Teledeltos" paper has the disadvantage of non-uniform electrical conductivity along the length of the roll and also with direction through the paper (anistropic).

The PAL analogue may be used for solutions of combined Poissonian and Laplacian fields, Poissonian fields or Laplacian fields. The two dimensional Laplacian current and potential patterns established on the analogue are analogous to a number of actual fields such as electrostatic, magnetostatic, electric current, temperature, fluid, etc. Examples of these analogies are shown in Figure 1.

\subsection{CONTINUOUS RESISTOR (PAL TYPE 1) ANALOGUE}

The circuit for the PAL Type 1 analogue is shown in Figure 2. This analogue consists of a resistive plotting surface, a coupled resistor, a direct current source, and a 
voltage measuring device. The cross sectional area of the problem with regard to geometry, physical properties, and boundary conditions is simulated by the resistive plotting surface. A voltage distribution across the plotting surface is directly analogous to the temperature distribution. A uniform current density enters the area of the plotting surface to simulate uniform heat generation. To accomplish this, a slab-type resistor of high electrical resistivity is coupled directly to the bottom of the plotting surface with a material that has an electrical resistivity of the same order of magnitude as that of the resistor. Since there is a voltage distribution across a portion of one surface of the resistor; namely, the voltage distribution across the plotting surface and none across the opposite surface, it is necessary that the voltage drop across the resistor be very large in comparison to the potential difference across the plotting surface. Also, to assure a uniform current density, current must be removed from the entire upper surface of the coupled resistor.

"Teledeltos" recording paper serves as a resistive plotting surface which can be readily cut to any geometric form desired. Equipotential (isothermal) boundaries are easily applied with silver paint. These boundaries serve as one terminal of the analogue. That part of the resistive plotting surface which represents the uniform heat-generating portion of the body is coupled to a resistor. The coupled resistor is a plate or slab of unfired "Lava" and the coupling is accomplished by gluing the "Teledeltos" paper to the "Lava" slab. The opposite side of the slab is coated with silver paint. This is the other terminal to which the leads from a direct-current source are attached. That portion of the upper surface of the slab-type resistor, which is not bonded to the conducting paper, is also coated with silver paint and it is connected into the circuit, through a variable resistor. A small non-silvered gap is left around the bonded area to insulate the conducting paper 
from the remainder of the upper surface. The variable resistor allows the potential difference across the non-silvered gap to a minimum. This is necessary to acquire a uniform current density per unit area throughout the slab.

When the circuit is set up in the manner described above, the current will enter the plotting surface, wherever it is bonded to the slab, with a uniform current density per unit area. The current will then flow through the conducting paper to the equipotential boundary. The current flow across the plotting surface creates the desired equipotential (isothermal) pattern which can be plotted by means of a null-type-voltage-measuring instrument.

Measurement of values directly proportional to temperature differences is possible if a so-called standard resistance is included in the circuit (Figure 1). The standard resistance is constructed from conducting paper. All the current that flows into the resistive plotting surface passes through the standard. By incorporating this standard in the circuit, the equation, relating temperature difference to the values of voltage measured on the analogue, can be written as (Appendix A)

$$
\Delta T=\left(Q_{T} / k_{t}\right)\left(\Delta E / \Delta E_{s t d}\right)
$$

when the standard resistance has a length to area ratio of one. Thus, to obtain a numerical value for the temperature difference between any two points in a body it is only necessary to multiply the value of $Q / k_{t}$ by the ratio of the voltage difference between the same two points on the analogue and the voltage difference across the standard.

The coupled resistor is made from unfired "Lava," which is a hydrous aluminum silicate. The electrical resistivity of this material is of the order of $10^{14} \mathrm{ohm}-\mathrm{cm}$ and is a function of its water content. 
Since it is necessary for accurate solutions that the coupled resistor deliver to the plotting surface an equal amount of current per each unit area, the resistor material must be as homogeneous as possible. This is especially true if a plot of the isotherms is desired. The size of the resistor in the PAL analogue is $15 \mathrm{in}$. by $15 \mathrm{in}$. by $1 / 4 \mathrm{in}$. thick.

Some of the errors involved in an analogue of this type are:

1. Non-uniformity of electrical conductivity of the conducting paper

2. Non-homogeneity of the coupled resistor

3. Voltage drop across the conducting surface

4. Variation in thickness of the mucilage

5. Difference in electrical conductivity of the standard from that of the plotting surface

6. Edge effects

7. Variation of the coupled resistor with humidity

8. Change of electrical conductivity of the conducting paper due to the application of mucilage

9. Dimensional errors in fabricating the analogue.

The two principle causes of error are non-uniformity of electrical conductivity of the conducting paper and non-homogeneity of the coupled resistor.

The error from non-uniform conductivity of the resistive plotting surface can be decreased if maximum use is made of symmetry. For example, consider a circularly distributed source; if only one-sixth of a circle is used instead of the entire circle, all the current will flow in approximately the same direction through the conducting paper. This will reduce 
the error caused by change of electrical conductivity of the paper with respect to direction of current flow.

The voltage drop across the resistive plotting surface is usually less than $0.001 \%$ of the voltage drop across the slab, thus, the error from this cause is negligible.

The thickness of the mucilage, which joins the conducting paper to the slabtype resistor, should be as uniform as possible. The electrical resistivity of the mucilage should be of the same order of magnitude as that of the coupled resistor. If the resistivity of the mucilage is too large, a substantial part of the voltage drop through the slab occurs across the glue. This leads to non-uniform current density caused by non-uniform thickness of the mucilage. If the mucilage is too good a conductor, it conducts a substantial portion of the current in parallel with the conducting paper and then temperature differences cannot be determined directly. Stationery mucilage operates satisfactorily.

A change in the resistance of the "Teledeltos" paper occurs upon application of the muci lage and varies with drying time. This error can be eliminated by applying the mucilage to both the standard and plotting surface at the same time. Dimensional errors are usually small since the paper can be cut accurately.

It is essential that the standard resistor and the resistive plotting surface be fabricated from the same vicinity of conducting paper since the electrical conductivity of the paper varies with position. It is also necessary that the standard resistor be coupled to a slab of "Lava" so that its unit resistance will approximate the resistance of the plotting surface. 
It is necessary for good results that the electrical flow lines through the coupled resistor be orthogonal to the surfaces. If they are other than orthogonal, edge effects are created in the plotting surface. This causes a distortion of the true voltage (temperature) pattern. Edge effects are minimized by painting with silver all of the upper surface of the resistor that is not coupled to the conducting paper. Of course, the silver paint on the upper surface must be insulated from the plotting surface. The silvered upper surface is connected through a variable resistor into the circuit as shown in Figure 2 and the potential difference between this surface and the periphery of the coupled portion of the plotting surface is adjusted to a minimum. The silver paint can be removed from the slab with acetone.

The electrical resistivity of "Lava" is greatly affected by changes of humidity. The over-all resistance of a slab may change many fold within a few hours, however, the absolute value of current flow through a slab is not important since at any particular time the current which flows into the plotting surface also flows through the standard resistance. Thus, the ratio of voltages which are used in the temperature-difference equation ( $E q, 1$ ) is constant with time. If a large humidity change occurs too rapidly, the flow distribution through the slab may be altered owing to local changes of resistivity。

The following problem demonstrates and establishes the accuracy of the PAL Type 1 analogue for a two dimensional problem. The problem deals with an infinitely long square bar ( $2 a$ by $2 a$ ) which is generating heat uniformly. The outer boundary is held at a constant temperature. The analytical solution to this problem is (Reference 1): 


$$
\Delta T=\frac{q^{\prime \prime \prime}\left(a^{2}-x^{2}\right)}{2 k}-\frac{q^{\prime \prime \prime} 16 a^{2}}{\pi^{3} k} \sum_{n=0}^{\infty} \frac{(-1)^{n} \cos [(2 n+1) \pi x / 2 a] \cosh [(2 n+1) \pi y / 2 a]}{(2 n+1)^{3} \cosh [(2 n+1) \pi / 2]}
$$

The pattern was constructed with the $y$ axis perpendicular to the rolled direction and the $x$ axis parallel to it. The standard resistance was fabricated standards cut in directions $90^{\circ}$ apart and connected into the circuit in parallel. The analytical and analogue results for traverses from one corner and both midpoints of the wall to the midpoint of the rod are shown in Figure 3. The difference between the analytical and analogue results at the hot spot is 4 per cent.

\subsection{LUMPED RESISTOR (PAL TYPE 2) ANALOGUE}

The circuit for the PAL Type 2 analogue is shown in Figure 4. This analogue consists of a resistive plotting surface, coupled $1 / 2$ watt resistors, a direct current source and a null-type voltage measuring instrument. The resistive plotting surface ("Teledeltos") simulates the problem with regard to geometry, physical properties, and boundary conditions.

In those areas of the plotting surface where heat generation is to be simulated, electric current is introduced into the paper at numerous points. This analogue varies from the previous one only by the method of introducing current into the plotting surface. The previous method utilized a slab-type resistor with some area of the plotting surface bonded to the slab whereas this method uses individual, 1/2 watt, commercially available resistors directly coupled to the plotting surface in a specified matrix. The electric current, associated with a particular area of the plotting surface, enters that area at a point, whereas in the former method it entered continuously over the entire area. Thus, the continuous resistortype analogue, for a sufficiently fine mesh grid, the electrical flow pattern in the resistive 
plotting surface very closely represents the true heat-flow pattern and only a small error occurs due to lumping the entering electric current at grid points. The current, which enters the conducting paper, passes across and leaves the plotting surface at one of the equipotential (isothermal) boundaries, passes through a standard resistance and returns to the battery. The values of temperature difference are determined by the equation

$$
\Delta T=\left(Q_{T} / k_{t}\right)\left(\Delta E / \Delta E_{s t d}\right)
$$

The construction of an analogue of this type requires numerous electrical connections which must be made accurately, rapidly, and simply. This may be done by using a specially designed "grid-board."

The grid-board eliminates innumerable wires and connectors that would otherwise be required for this type of analogue. The grid-board is made from a slab of Lucite, approximately $36 \mathrm{in}$. by $36 \mathrm{in}$. by $3 / 4 \mathrm{in.}$. A matrix of resistor positions of the dimensions given in Figure 10 is located in the central section of the board. The matrix may be of any geometry - square, triangular, etc. The author used a $1 / 2$ in. by $1 / 2$ in. square matrix over an area $18 \mathrm{in}$. by $18 \mathrm{in}$. On the underside of the board each row of the matrix is covered by a solid, rectangular, brass bus bar that is held firmly against the Lucite and resistors.

In order to operate properly in the grid-board, the resistor leads are modified. One lead, or "pigtail," is cut off as short as is practical and the other is cut off so that it protrudes $1 / 8$ in. above the upper surface of the board when the resistor is in working position. The long lead is sharpened to facilitate puncturing the conducting paper. 
When inserting a resistor into position in the grid-board, a small spring is placed around the long resistor lead and inserted into position in the grid-board along with the resistor. During this operation the long lead punctures the plotting surface which has previously been properly positioned on the upper surface of the grid-board. When all resistors and springs are in place, the brass bus bars are fastened firmly against the grid-board. The small springs hold the short resistor leads securely against the bus bars, making a positive electrical connection. A small drop of silver paint is carefully applied to the end of each long resistor lead. The silver paint runs down the lead and makes a firm connection between the lead and the conducting paper.

The possible sources of errors in this method are:

1. Non-uniformity of electrical conductivity of the conducting paper

2. Variation of resistance between individual resistors

3. Voltage drop across the plotting surface

4. Disturbance of the electrical flow pattern in the plotting surface caused by the electrical connection of a resistor at each grid position

5. Difference in electrical conductivity of the standard from that of the plotting surface

6. Approximation of a boundary by a matrix

7. Faulty bus bar connections

8. Dimensional errors in fabricating the analogue.

Items 1, 3, 5 and 8 have been discussed previously and all previous conclusions apply equally as well to this method. 
The variation of resistance between individual resistors (Item 2) can be held to as close a tolerance as is required; therefore, this source of error can be made negligible. At each grid position there is a disturbance of the desired flow pattern. The potential of the resistor lead is slightly higher than the plotting surface immediately surrounding it. Thus it is necessary to avoid making any measurements at these points. The preferred measurement positions are midway between grid points.

The resistors are $1 / 2$ watt, $5 \%$ tolerance, fixed-composition resistors that are commercially avai lable. For uniform heat-generation problems, where all resistors are of the same value, the absolute value of resistance of each resistor is not as important as the uniformity of values.

In order to insure the proper electrical flow pattern, the voltage drop across each resistor must be very nearly the same. Since the resistors are connected in parallel between the conducting paper and the bus bars, the voltage drop across the plotting surface must be small (less than 1\%) compared to the voltage drop across the resistors proper. This condition imposes a limitation upon the maximum number of grid positions that can be used for any specific problem.

Since the minimum physical dimensions of the matrix is fixed when a gridboard is used, it is necessary to apply a scale factor to the model. The proper scale factor depends upon the complexity of the boundaries - the more complex the boundaries are, the finer should the matrix be. It is necessary, however, to keep in mind that for any given geometric configuration and fixed maximum resistors, there is a maximum number of grid positions that can be used without introducing sizeable errors from the voltage drop across the plotting surface. 
Faulty bus bar connections are always a potential hazard. The hazard may be practically eliminated by carefully checking each connection.

The following problem demonstrates the importance of constructing the proper standard resistor and establishing the accuracy of the analogue for two dimensional problems. This problem deals with an infinitely long square bar (2a by $2 a$ ) which is generating heat uniformly. The outer surface is held at a constant temperature. The analytical solution to this problem is equation 2.

The pattern was constructed with $y$ axis perpendicular to the rolled direction and the $x$ axis parallel to it. Three different standard resistors were used in determining the temperature distribution of the square bar. The first standard was constructed so that the current flow across the standard was perpendicular to the rolled direction, the second standard was constructed so that the current flow was parallel to the rolled direction of the "Teledeltos" paper, and the third standard was made up of the first two wired in parallel.

The analytical results along with the PAL analogue results for the three standard resistors are plotted in Figures 5, 6 and 7. The error at the hot spot is 3.5 per cent for the first standard, 8.7 per cent for the second standard and 2.6 per cent for the third standard. The results using the third standard also produce greater accuracy for other locations in the square bar.

Variable heat generation with respect to geometry as well as uniform heatgeneration problems can be solved with this analogue by proportioning the electrical flow into the plotting surface according to the same geometric pattern as the heat-generation pattern. 
This is done by using the proper value of voltage at each grid position, since the current flow into any grid position is proportional to the voltage at that point. Thus the only requirement for the solution of a variable heat-generation problem above those already specified is a knowledge of the heat generation as a function of geometry. This method approximates the continuous varying heat generation by finite steps.

The results of a variable heat-generation problem is shown in Figure 8 . The problem is an infinite plate with the following boundary conditions

$$
\begin{array}{rrr}
x=0 & T=T_{0} \\
x=1 / 2 & \partial T / \partial x=0
\end{array}
$$

where

$$
q^{\prime \prime \prime}=q_{0}^{\prime \prime \prime}(1+2 x)
$$

The temperature difference function through the plate is

$$
\frac{\Delta T}{q_{0}^{\prime \prime \prime} / k}=\frac{1 x}{2}-\frac{x^{2}}{2}+\frac{1^{2} x}{4}-\frac{x^{3}}{3}
$$

The error at the maximum temperature difference is approximately 1.7 per cent of the temperature difference. The percentage error is greater close to the surface of the plate. This is usually advantageous since the maximum temperature difference is generally the most critical.

\subsection{STANDARD RESISTANCE}

A standard resistance constructed from conducting paper is included in the circuit of each of the PAL analogues described in this report. This standard resistance serves a variety of purposes. 
When a standard is used, no conditions are imposed on the quantity of current flowing in the system.

It allows a numerical value of temperature difference between any two points on the plotting surface to be determined directly from the ratio of the voltage difference between these same two points and the voltage difference across the standard (see Appendix A).

The use of a standard resistance practically eliminates the error introduced by the variation of unit resistance of the paper with humidity. The resistance of the analogue and the standard are both affected in the same manner so long as they are used under the same ambient conditions. Therefore, the ratio of voltage drop across some part of the resistive plotting surface to that across the standard will be affected to a negligible extent if the humidity change is slow. The standard, however, will not eliminate local effects. Thus, it is important that the plotting surface should not be touched. Also, a local change of resistance will cause a local error in the isothermal line position.

The error caused by the non-uniform electrical conductivity of the paper is considerably reduced by proper construction of the standard.

The standard should be fabricated so that the current flow through it is oriented in the same direction with respect to the direction along the roll, as the major flow of current in the resistive plotting surface. If there is no major direction of current flow in the resistive plotting surface, then two standards with area-to-length ratios of $1 / 2$ are cut in directions $90^{\circ}$ apart and connected into the circuit in parallel. This gives a potential difference across the standard that is more nearly correct. 


$$
\Delta T=\frac{q_{T}^{\prime}}{k_{t}} \frac{\Delta E}{\Delta E_{s t d}}\left(\frac{L}{A}\right)_{s t d}
$$

where the length-to-area ratio of the standard is determined for the particular shape of standard being used.

\subsection{DIFFERENCE IN THERMAL CONDUCTIVITY BETWEEN REGIONS}

A difference in thermal conductivity between regions is accomplished by utilizing one or more parallel electrical flow paths. In this manner simple ratios of thermal conductivities between regions are created. When fabricating the analogue, additional pieces of conducting paper are cut the exact shape as those areas of the plotting surface that represent the higher thermal conductivity material. These sheets of conducting paper are electrically connected to those areas of the plotting surface by the application of silver paint at numerous points around their periphery. This creates parallel electrical flow paths of the same identical shape for those portions of the plotting surface where higher thermal conductivity material is to be simulated. Across the regions of multiple layers of conducting paper the voltage drop is decreased by a factor proportional to the number of parallel paths created, and the potential measurements represent the desired problem. There is, of course, a practical limitation to the number of parallel paths that can be accurately constructed in this manner.

In the case of the continuous resistor-type analogue, the area of the plotting surface, where heat generation is to be simulated, is bonded to the "Lava" slab. Therefore, it is necessary that only one layer of conducting paper be used for that portion of the plotting surface. This limitation restricts this method to problems where the regions surrounding the 
heat-generation areas have a thermal conductivity equal to or greater than that of the heatgenerating regions.

This restriction does not apply to the lumped resistor-type analogue since many parallel flow paths can be created in the heat-generating region.

\subsection{NON-ISOTHERMAL BOUNDARY CONDITIONS (HEAT TRANSFER FROM A SURFACE)}

Heat transfer from a surface to an isothermal fluid is simulated by adding an additional resistance to the electrical current that flows out of the area of the analogue which simulates the heat transfer surface. The additional resistance is created by extending the plotting surface in a direction perpendicular to the line representing the heat transfer surface by a length equal to the ratio $\beta k / h$. This length of paper is slit a sufficient number of times in a direction orthogonal to the line representing the heat transfer surface to insure a small voltage drop across any individual strip as compared to the voltage drop along the length of the strip. If it should be necessary to extend the plotting surface in a direction other than perpendicular, then the length of this extension should be adjusted for the change in electrical flow area as compared to the area available for electrical flow when the strips are orthogonal. Whenever heat transfer from a surface is involved, the isothermal boundary is the fluid temperature.

\subsection{CONCLUSIONS}

The analogue techniques described in this report are particularly applicable to steady-state, multi-region problems with internal heat generation, and wherein the ratios of thermal conductivities between regions are small integers and the heat generation is a function of geometry only. Therefore, these methods of solving heat transfer in oddly shaped 
bodies should be useful tools since by these techniques solutions to difficult problems are readily obtained at a minimum of time and expense.

The principle advantage of these methods is the ease with which complex geometries can be handled. The "Teledeltos" paper, which is used as the conducting medium, can be readily formed into complicated shapes with simple tools and a minimum of effort. Furthermore, since the conducting paper is a continuous medium, the electrical flow is more nearly analogous to heat flow than in a comparable lumped circuit type analogue or numerical analogy.

Almost invariably, heat-generating bodies dissipate their energy to a flowing fluid. These analogues can readily accommodate boundary conditions of this sort if the heat transfer coefficient is known as a function of geometry. This is due to the fact that heat flow from a surface to a single phase liquid can be expressed by a conduction-type equation.

The chief difficulty in using these methods is the error caused by the directional properties of the conducting paper. Normally this error is compensated by proper construction of the standard.

The principle difference between the two techniques described in this report is the method of introducing the electrical current into the conducting paper. The continuous resistor-type analogue has one principle advantage over the lumped-resistor type, that is, for a given size analogue a more complex geometry can be handled with an acceptable degree of accuracy. Otherwise, the lumped-resistor technique is superior because of the ease in which the problem can be set up. 


\subsection{REFERENCES}

1. H. S. Carslaw and J. C. Jaeger, Conduction of Heat in Solids, Oxford University Press, 1947.

2. "Use and Theory of the Analogue Field Plotter," Sunshine Scientific Instruments, Philadelphia, Pennsylvania, IM24.

3. W. R. Simmons, "Electrical, Geometrical Analogue Techniques for the Solution of Twodirectional Complex Heat Conduction Problems," Argonne National Laboratory, Lemont, Illinois, ANL - 5319. 


\section{APPENDIX A}

\section{DERIVATION OF THE TEMPERATURE DIFFERENCE EQUATION}

The equation for the temperature difference between any two points is derived in the following manner. Consider a body generating heat where the heat generation as a function of position is known.

$$
q^{\prime \prime \prime} \quad=f(\alpha, \rho)
$$

Assume the PAL analogue is set up to simulate the heat generation in the body such that

$$
i^{m} \quad=f(\beta a, \beta \rho)
$$

where $\beta$ is the scale factor. Then the solution of the Poisson equation

$$
\nabla^{2} \mathrm{~T}=-q^{\prime \prime \prime} / k_{t}
$$

will be the same as

$$
\text { then } \quad \begin{aligned}
\nabla^{2} E & =-i{ }^{\prime \prime} / k_{e} \\
\text { and } \quad & =1 / k_{t} F(\alpha, \rho)+c_{1} \\
k_{t} \frac{\partial T}{\partial n} & =\frac{\partial T}{\partial \alpha} \frac{\partial \alpha}{\partial n}+\frac{\partial T}{\partial \rho} \frac{\partial \rho}{\partial n} \\
k_{e} \frac{\partial E}{\partial n} & =\frac{\partial E}{\partial \alpha} \frac{\partial \alpha}{\partial n}+\frac{\partial E}{\partial \rho} \frac{\partial \rho}{\partial n} \\
k_{t} \frac{\partial T}{\partial n} & =\frac{\partial F}{\partial \alpha} \frac{\partial \alpha}{\partial n}+\frac{\partial F}{\partial P} \frac{\partial \rho}{\partial n} \\
k_{e} \frac{\partial E}{\partial n} & =\frac{1}{\beta}
\end{aligned}
$$


The preceding equation is then equal to a constant at any point $\alpha_{0}, P_{0}$. At some point $\alpha_{1}, P_{1}$ there is a quantity of heat flux $q$, where

$$
q=k_{t}\left(\frac{d T}{d n}\right)_{a_{1}, P_{1}}
$$

At an equivalent point $\beta \alpha, \beta$, on the analogue plotting surface, there is a quantity of current flux, $i$, where

$$
i \quad=-k_{e}\left(\frac{d E}{d n}\right)_{\beta \alpha_{1}, \beta} \rho_{1}
$$

Combining equations 10 and 11

$$
\frac{q}{i}=\frac{k_{t} \frac{d T}{d n}}{k_{e} \frac{d E}{d n}}
$$

Converting to finite difference and rearranging

$$
\Delta T=\frac{q}{i} \frac{k_{e}}{k_{t}} \Delta E
$$

from equations 9 and 12

$$
\frac{q}{i}=\frac{1}{\beta}
$$

and

$$
\beta k_{t} \frac{\partial T}{\partial n}=k_{e} \frac{\partial E}{\partial n}
$$

integrating the flux on the boundary $S$ where $n$ is normal to the boundary

$$
\beta k_{t} \int_{s} \frac{\partial T}{\partial n} d s=k_{e} \int_{s} \frac{\partial E}{\partial n} d s
$$


then $\quad \beta Q_{T}=I_{T}$

The total current which flows into the analogue also flows through the standard resistance and can be written

$$
\mathrm{I}_{\mathrm{T}}=\mathrm{k}_{\mathrm{e}}\left(\frac{\mathrm{A}}{\mathrm{L}}\right) \Delta \mathrm{E}_{\text {std }}
$$

let

$$
\frac{\mathrm{A}}{\mathrm{L}}=1
$$

therefore

$$
\frac{q}{i}=\frac{1}{\beta}=\frac{Q_{T}}{T_{T}}=\frac{Q_{T}}{k_{e} \Delta E_{s t d}}
$$

Substituting equation 19 into equation 13 we have

$$
\Delta T=\frac{Q_{T} \Delta E}{k_{e} \Delta E_{s t d}}
$$

From equation 20 only two measurements are necessary to obtain a temperature difference. 
APPENDIX B

TEMPERATURE DIFFERENCE EQUATION FOR AN INFINITE PLANE PLATE

WITH A LINEAR VARIATION IN HEAT GENERATION

Poissons equation for linear variation in heat generation is:

$$
\frac{d^{2} T}{d x^{2}}=-q^{\prime \prime \prime} / k=-\frac{q_{0}^{\prime \prime \prime}(1+2 x)}{k}
$$

Integrating

$$
\begin{aligned}
& \frac{d T}{d x} \quad=-\frac{q_{0}^{\prime \prime \prime}}{k}\left(x+x^{2}+c_{1}\right) \\
& T \quad=-\frac{q_{0}^{\prime \prime \prime}}{k}\left(\frac{x^{2}}{2}+\frac{x^{3}}{3}+c_{1} x\right)+c_{2}
\end{aligned}
$$

For the following boundary conditions

$$
\begin{array}{ll}
x=0 & T=T_{0} \\
x=1 / 2 & \frac{\partial T}{\partial x}=0 \\
T \quad=\frac{q_{0}^{\prime \prime \prime}}{k}\left(\frac{1 x}{2}-\frac{x^{2}}{2}+\frac{1^{2} x}{4}-\frac{x^{3}}{3}\right)+T_{0}
\end{array}
$$

then

$$
\frac{\Delta T}{q_{0}^{\prime \prime \prime} / k}=\frac{1 x}{2}-\frac{x^{2}}{2}+\frac{1^{2} x}{4}-\frac{x^{3}}{3}
$$




\section{APPENDIX C}

(1)

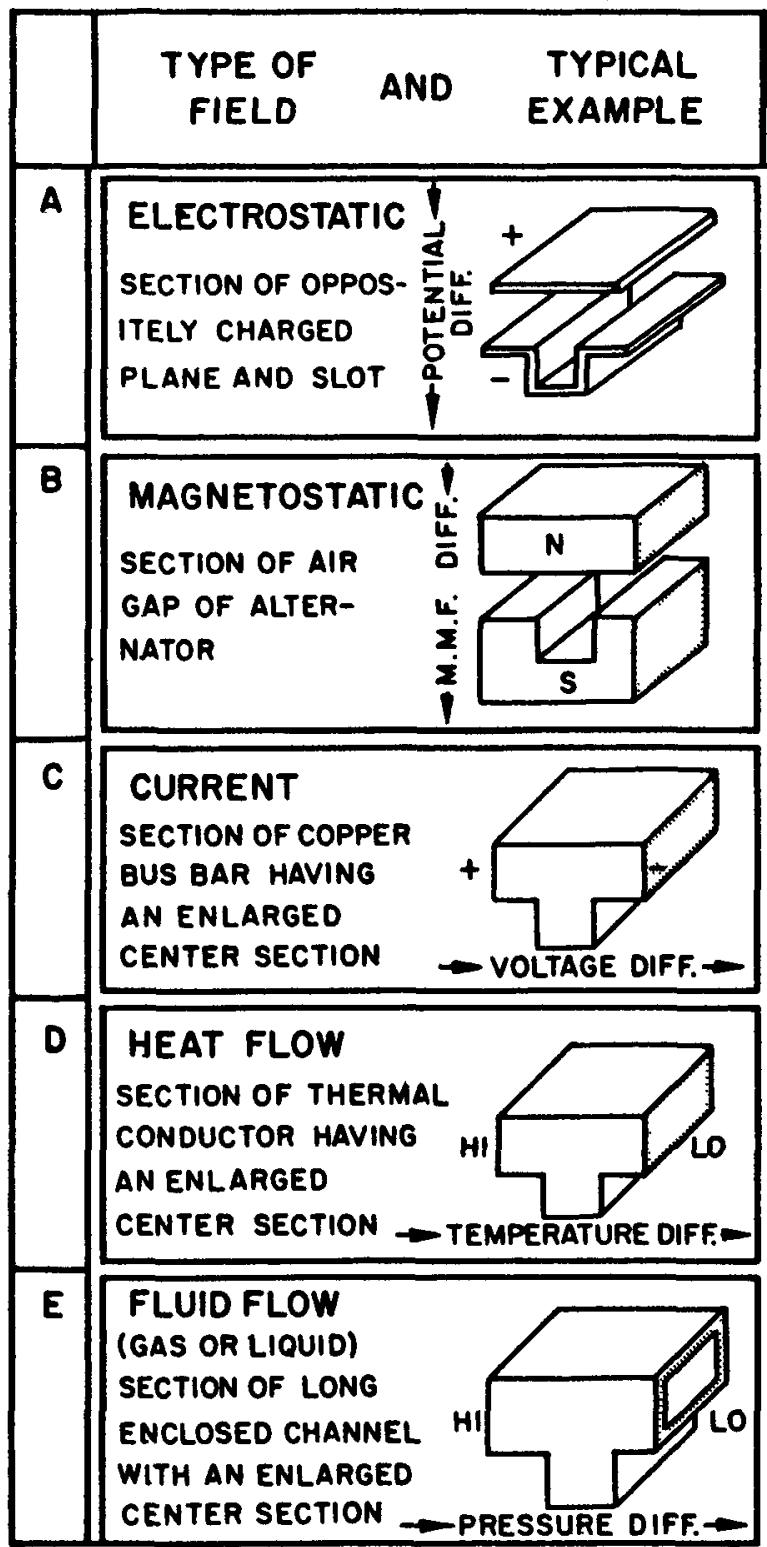

(3)

\begin{tabular}{l|l|l|}
\hline SHOWING IOENTI- & SHOWING IDENTI- & SHOWING IDENTICAL \\
CAL FLUX OR & CAL EQUIPOTEN- & "COMBINED" ORTHO- \\
FLOW LINE & TIAL LINE & GONAL FIELD \\
PATTERNS & PATTERNS & PATTERN $[(3)+(4)]$ \\
\hline
\end{tabular}
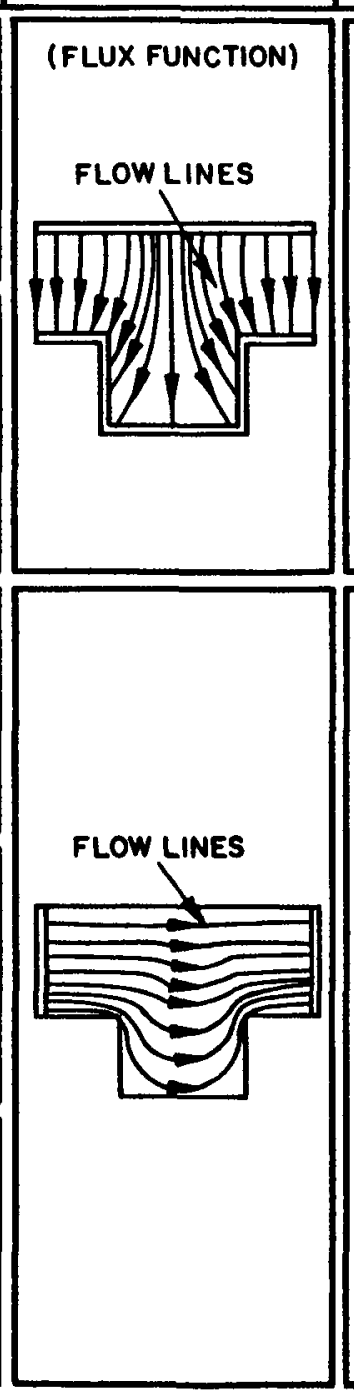

(4)

(5)
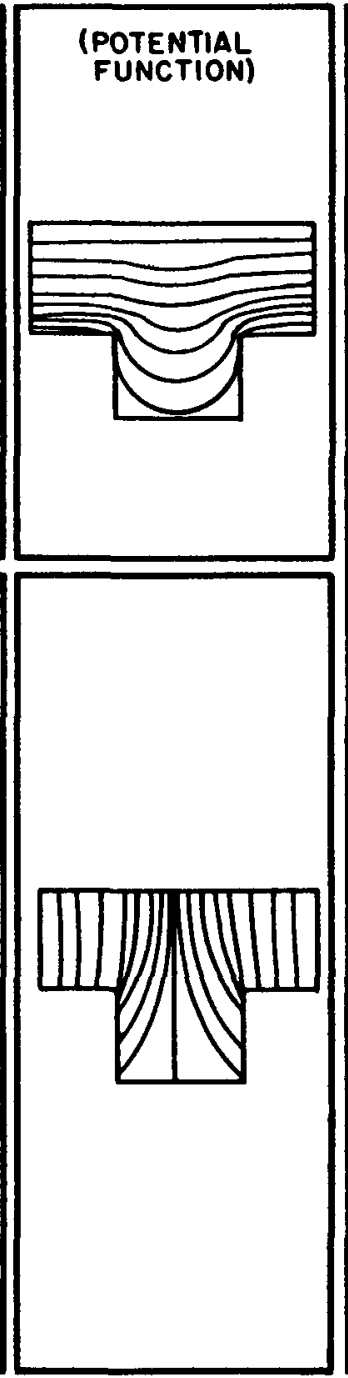

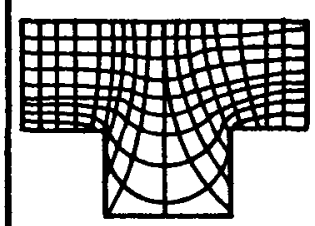

FIGURE I

EXAMPLES OF ANALOGIES BETWEEN PROBLEMS IN FIVE DIFFERENT TYPES OF LAPLACIAN FIELDS 


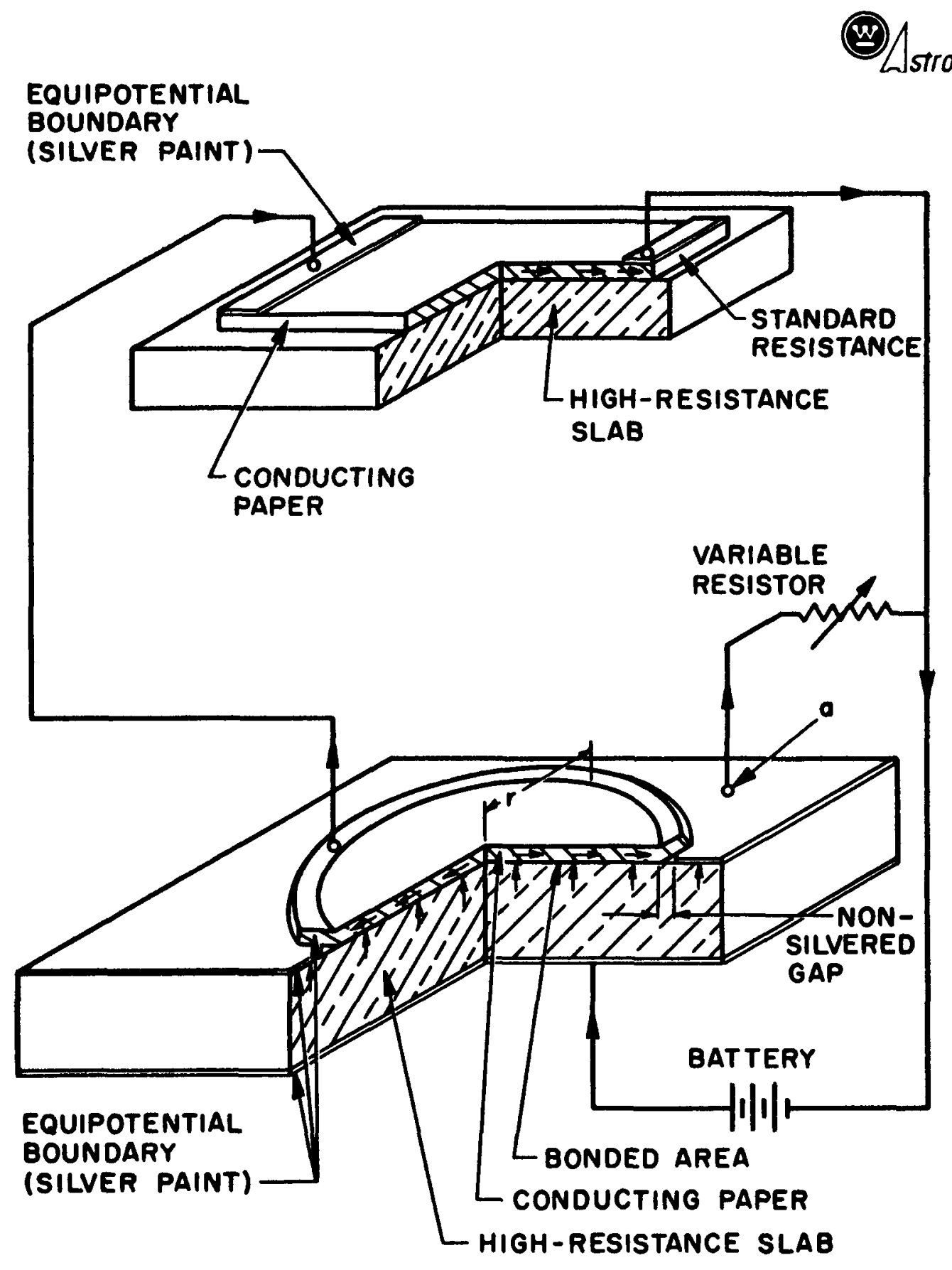

FIGURE 2

CIRCUIT DIAGRAM OF A CONTINUOUS RESISTOR (PAL ANALOGUE TYPE I) ANALOGUE 


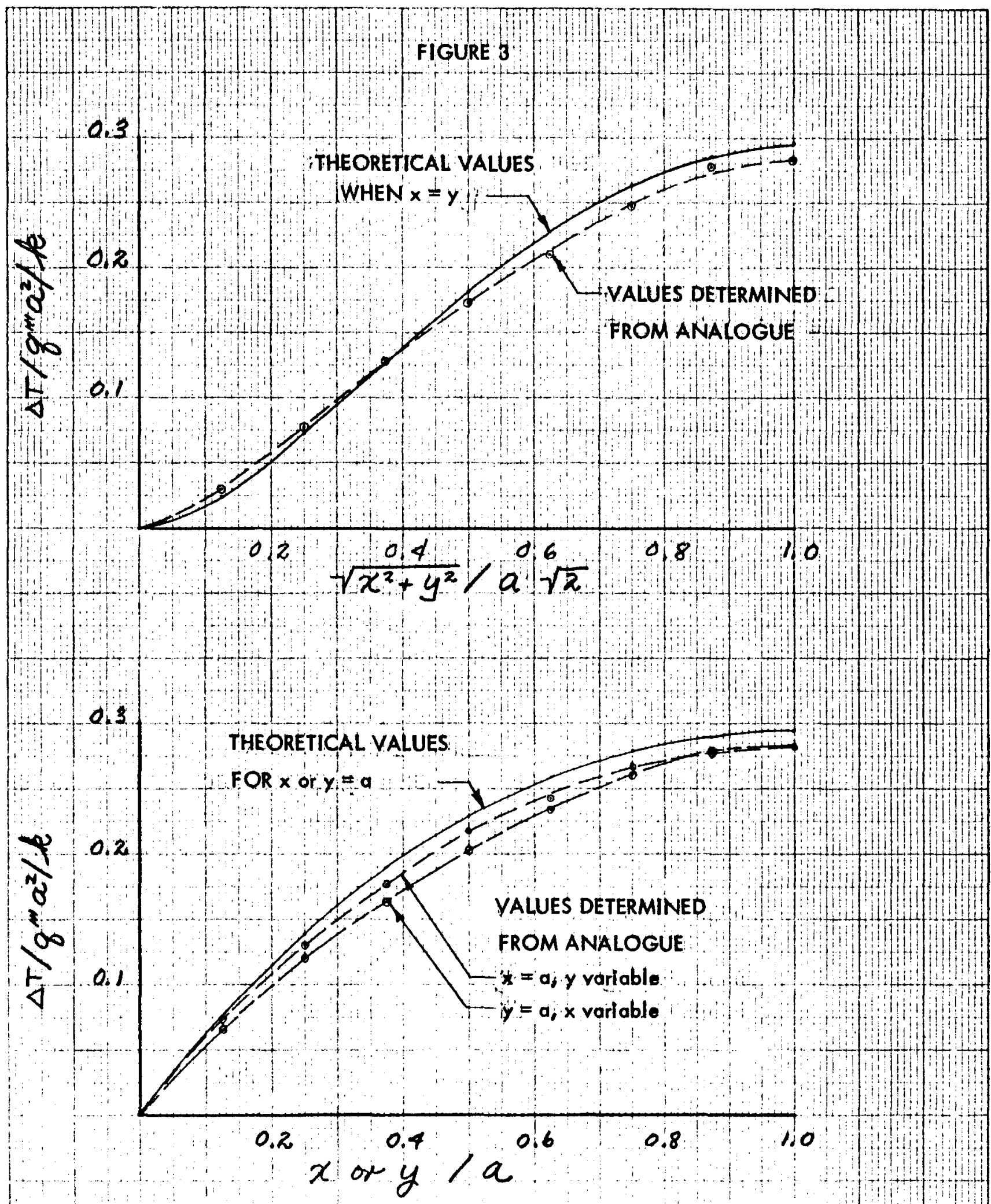

TEMPERATURE DIFFERENCE FUNCTION PROFILE IN A SQUARE ROD $(2 a \times 2 a)$ WITH UNIFORM HEAT GENERATION (PAL ANALOGUE TYPE 1) 


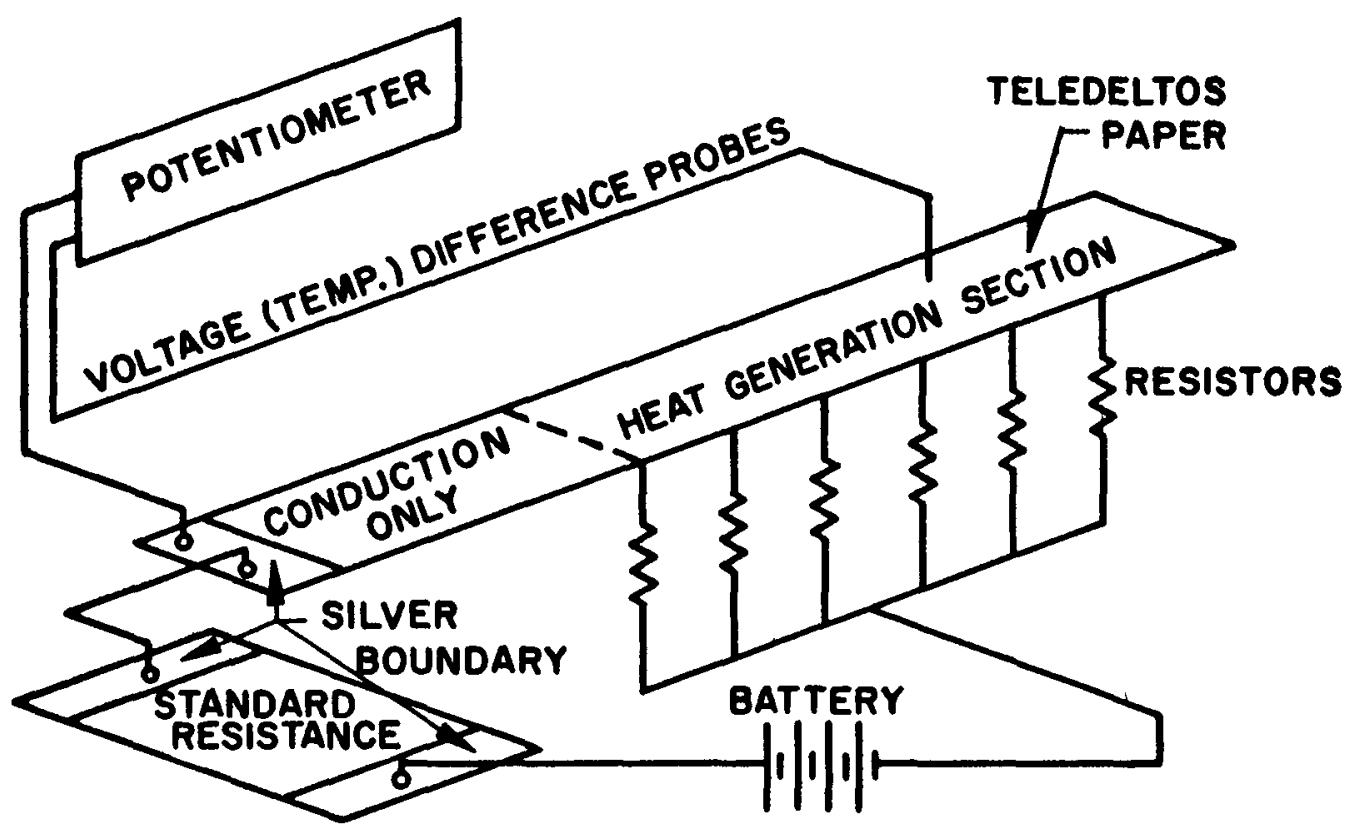

FIGURE 4

CIRCUIT DIAGRAM OF A LUMPED RESISTOR (PAL ANALOGUE TYPE 2) ANALOGUE 


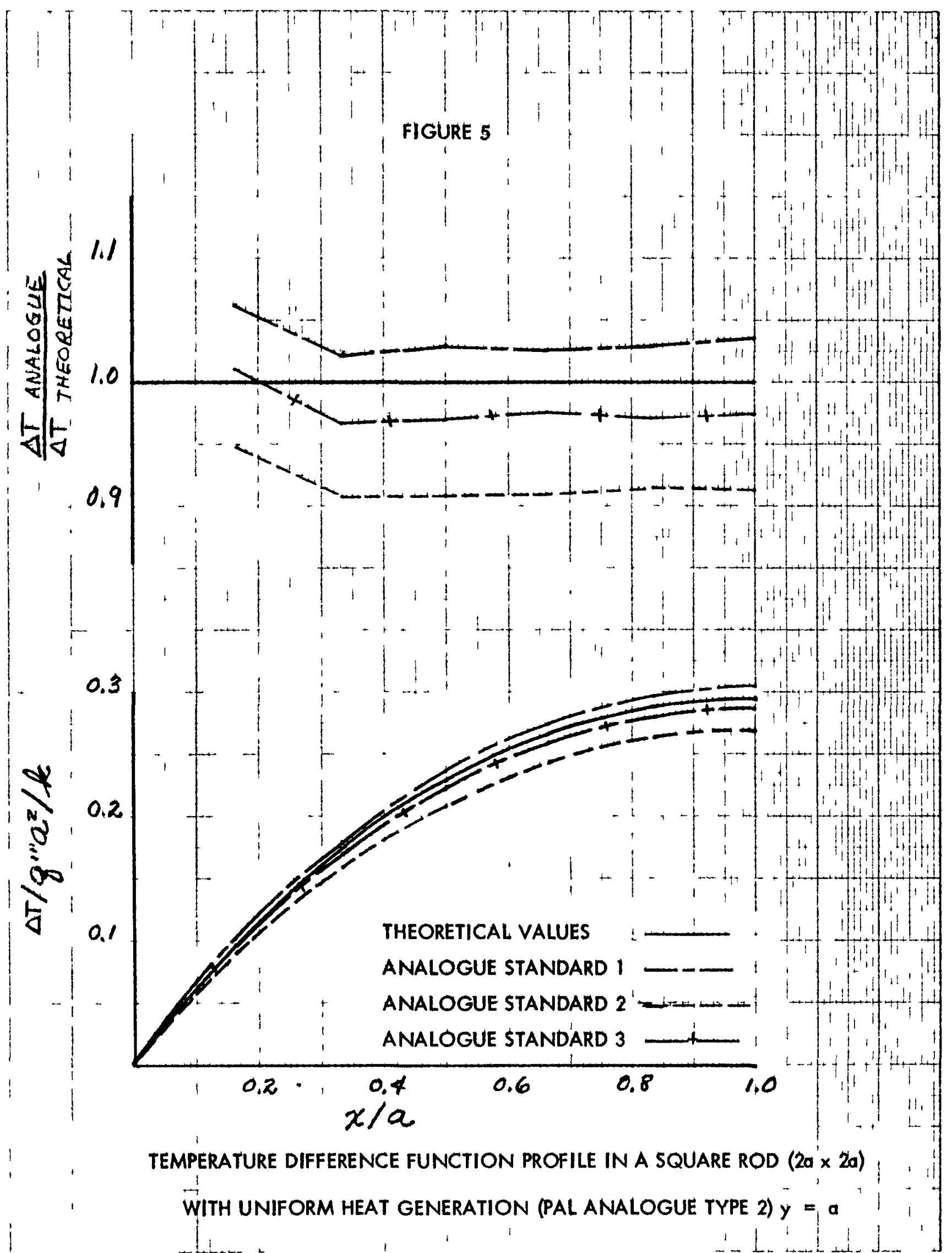




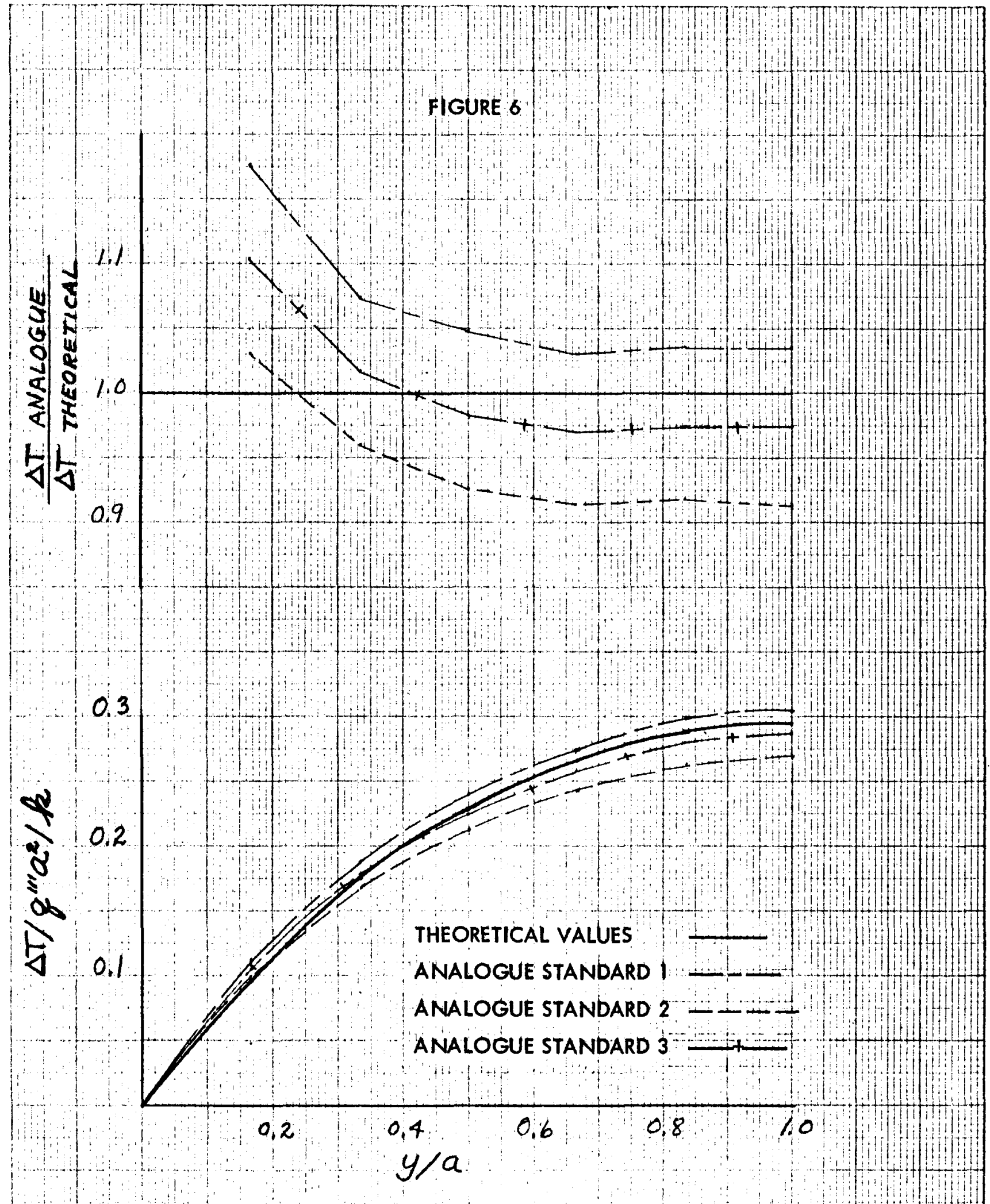

TEMPERATURE DIFFERENCE FUNCTION PROFILE IN A SQUARE ROD $(2 a \times 20)$ WITH UNIFORM HEAT GENERATION (PAL ANALOGUE TYPE 2$) x=0$ 


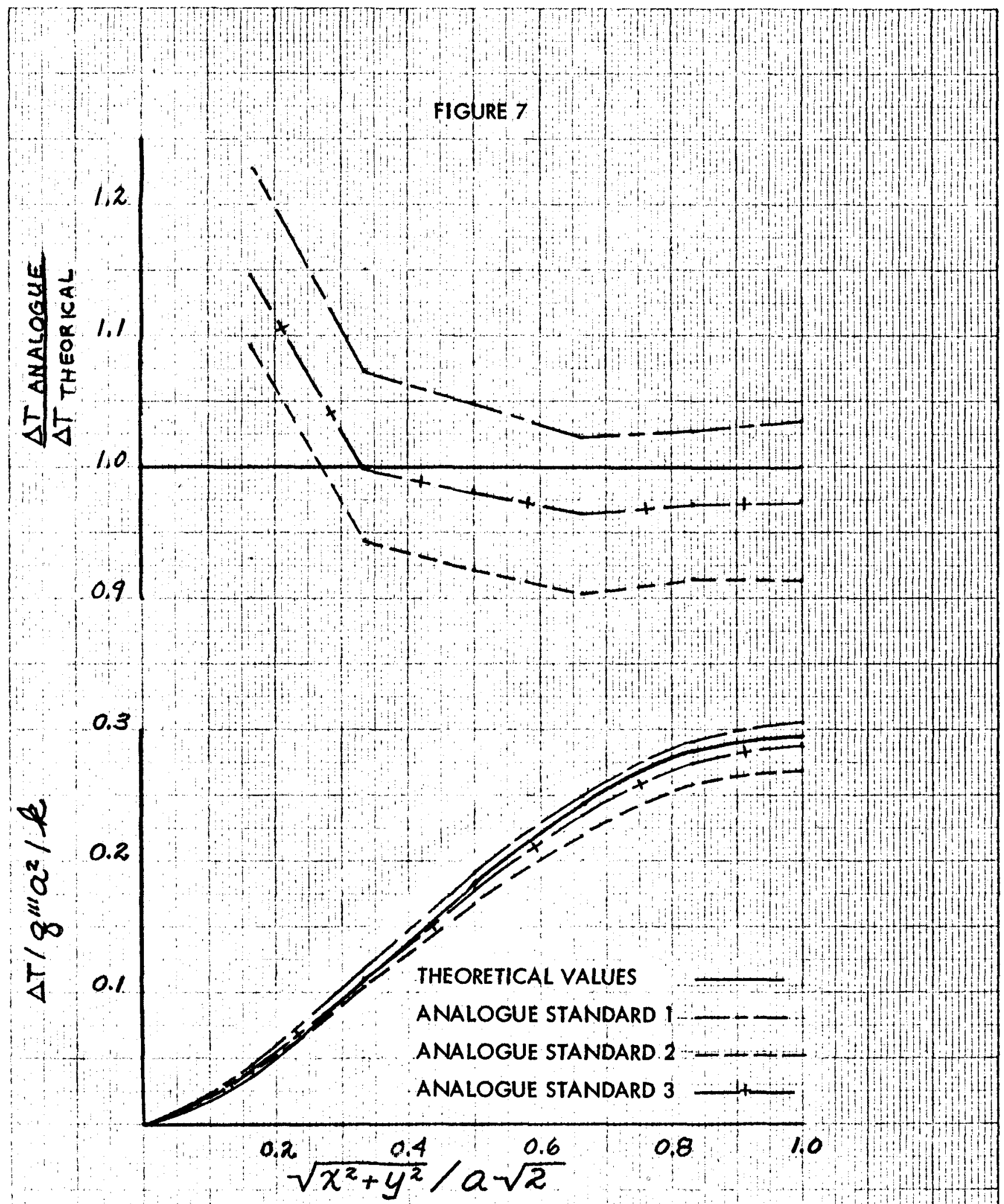

TEMPERATURE DIFFERENCE FUNCTION PROFILE IN A SQUARE ROD ( $2 a \times 2 a)$ WITH UNIFORM HEAT GENERATION (PAL ANALOGUE TYPE 2) $x=y$ 


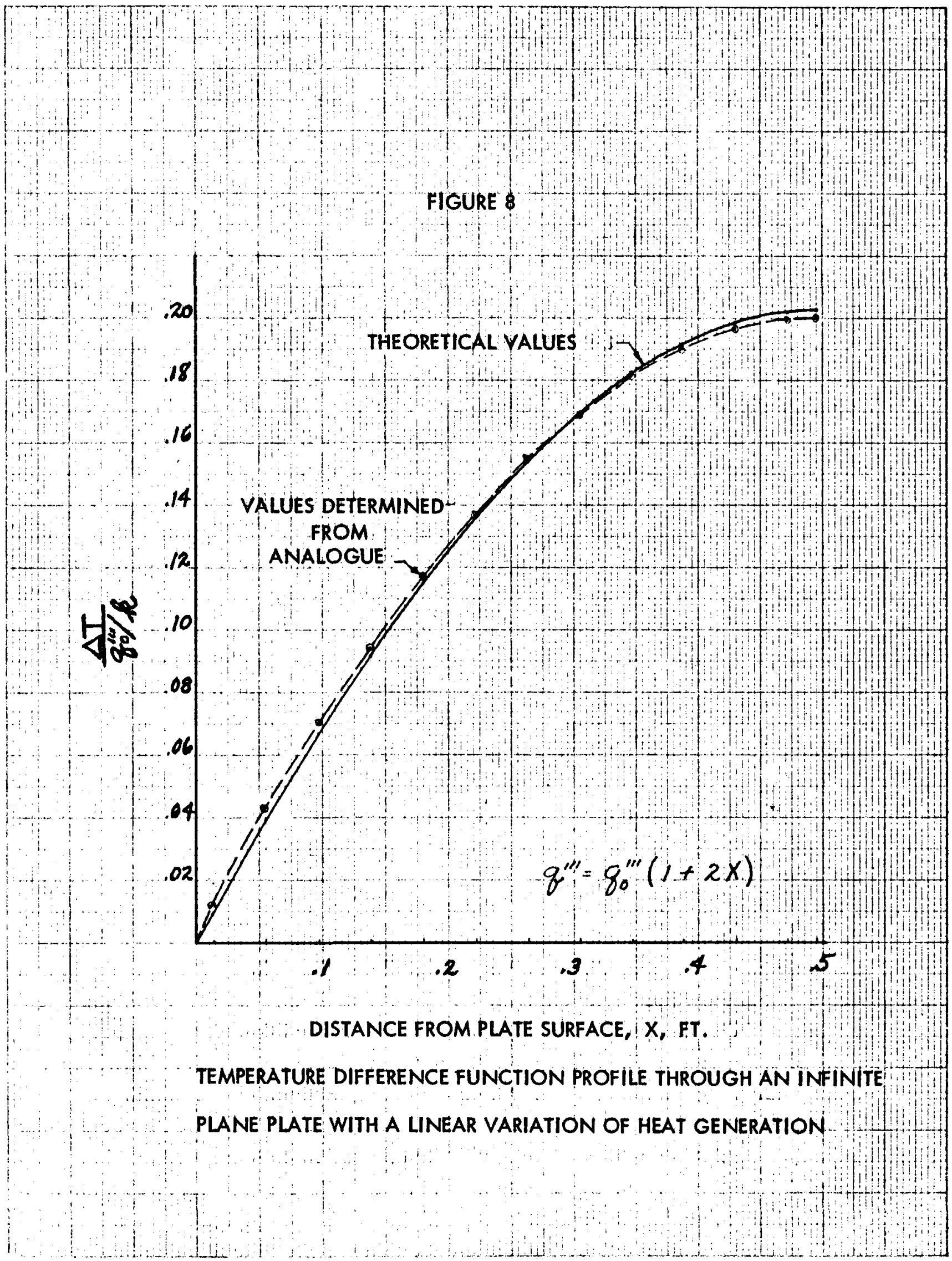

